\title{
Turbulent Convection: A New Model
}

\author{
V. M. Canuto \\ NASA Goddard Space Flight Center \\ Institute for Space Studies \\ 2880 Broadway \\ New York, NY 10025
}

\begin{abstract}
We use the latest models of turbulence to compute a new expression for the turbulent convective flux, $F_{c}$. The new values of $F_{c}$ are up to ten times larger than those given by the mixing length theory, MLT. Astrophysical considerations indicate that the new model fares better with observational data than the MLT.
\end{abstract}

\section{Introduction}

In stellar interiors, a great deal of heat is transported by convection rather than by radiation. Consequently, a reliable model for the convective heat flux $F_{c}$ is needed. The model adopted thus far in astrophysics, the mixing length theory, MLT (Böhm-Vitense, 1958), can yield results in agreement with the data provided the expression for $F_{c}(\mathrm{MLT})$ is increased by an arbitrary amount $\alpha>1$ which is not unique for all stars. The situation is clearly unsatisfactory.

To construct a more reliable model for convective turbulence one must: 1) forsake the intuitive physical picture of the MLT and couch the problem in terms of modern turbulence theory, 2) specify the exact nature of the approximations underlying the MLT, and, finally, 3) begin a systematic removal of these approximations. We have initiated this program and have found that MLT model is based on the following assumptions:

(a) Whereas the turbulent kinetic energy is known to be distributed among eddies of all sizes, the MLT takes into account only one eddy that contains all of this energy. That is, the MLT assumes an eddy energy spectrum $E(k)$ of the form

$$
E(k) \propto \delta\left(k-k_{0}\right),
$$

centered around the smallest wavenumber $k_{0}$ corresponding to the largest eddy compatible with the geometry of the system. (The integral of $E(k)$ over all $k$ yields the turbulent kinetic energy per unit mass.)

(b) The basic difficulty of turbulence is the non-linear term (NLT) that distributes the energy over a wide range of eddies. Although the net action of the NLT on a given eddy is in general a complex set of processes, the two main effects are cascade and backscatter: given an eddy of size $\sim 1 / k$, the effect of all the smaller eddies is to remove energy from it (cascade), as well as to return some of that energy (backscatter), i.e., schematically we have 


$$
\text { NLT }=\text { Cascade }+ \text { Backscatter }
$$

The MLT is equivalent to taking

$$
\text { NLT }=\text { Cascade, }
$$

an approximation that clearly underestimates the convective flux.

(c) The nonlinear interactions operate on time scales that depend on the size of the eddies, i.e., the eddy correlation time scale $1 / n_{c}(k)$ is a strong function of $k$ : eddies with dimensions comparable with the size of the system are dominated primarily by the stirring process, while medium size eddies resulting from repeated break-up processes have lost memory of the nature of the source and have a correlation time scale independent of the source. We have found that the MLT assumes that

$$
n_{c}(k) \sim n_{s}(k),
$$

where $1 / n_{g}(k)$ is the time scale characterizing the instability of the source. It is clear that Eq. (4) is true for a limited group of large eddies only.

We shall remove approximations (a)-(c) by trading the MLT formalism in favor of a turbulence theory that has become available thanks to recent progress in turbulence modeling (DIA, Kraichnan, 1964; EDQNM, Lesieur, 1987; RNG, Yakhot and Orszag, 1986; GISS model, Canuto et al., 1985, 1987).

\section{A new derivation of the MLT flux}

Consider the Fourier transformed equations for the velocity and temperature fluctuations $u_{i}(\mathbf{k})$ and $\theta(\mathbf{k})$, where $i=1,2,3$ (Ledoux et al., 1961; Yamaguchi, 1963; Nakano et al., 1979). (The time dependence of $u_{i}$ and is omitted for brevity and the summation convention is adopted.)

$$
\begin{gathered}
\left(\frac{\partial}{\partial t}+\nu k^{2}\right) u_{i}(\boldsymbol{k})+\operatorname{NLT}_{1}(\boldsymbol{k})_{i}=g \alpha \lambda_{j} \Pi_{i j}(\boldsymbol{k}) \theta(\boldsymbol{k}), \\
\left(\frac{\partial}{\partial t}+\chi k^{2}\right) \theta(\boldsymbol{k})+\mathrm{NLT}_{2}(\boldsymbol{k})=\beta \lambda_{i} u_{i}(\boldsymbol{k}),
\end{gathered}
$$

where $\alpha=1 / T, \nu$ is the kinematic viscosity, $\chi$ is the thermometric conductivity (thermal conductivity $\left.K=c_{p} \rho \chi\right), \beta=T\left(\nabla-\nabla_{\mathrm{ad}}\right) / H_{p}$ is the superadiabatic temperature gradient and $\lambda=(0,0,1)$. The non-linear terms $\operatorname{NLT}(\boldsymbol{k})$ are defined as

$$
\begin{gathered}
\operatorname{NLT}_{1}(\boldsymbol{k})=\Lambda_{i n m}(\boldsymbol{k}) \sum_{\boldsymbol{q}} u_{n}(\boldsymbol{k}-\boldsymbol{q}) u_{m}(\boldsymbol{q}), \\
\operatorname{NLT}_{2}(\boldsymbol{k})=i k_{n} \sum_{\boldsymbol{q}} u(\boldsymbol{k}-\boldsymbol{q}) \theta(\boldsymbol{q}),
\end{gathered}
$$

where $\Pi_{i j}=\delta_{i j}-k_{i} k_{j} / k^{2}$ and $2 \Lambda_{i n m}=i\left(k_{n} \Pi_{i m}+k_{m} \Pi_{i n}\right)$.

The most important terms are the non-linear terms which couple velocity fields $u_{i}(k)$ with different wavenumbers, i.e., eddies of different sizes, and thus determine 
the resulting energy spectrum of the turbulent eddies. The reliability of any turbulence model depends crucially on how successfully the non-linear terms are treated. Before discussing that, however, there is a basic physical property of the NLT that must be stressed. Suppose one assumes that $\operatorname{NLT}(\boldsymbol{k})=0$. In this case, there cannot be a stationary solution of Eqs. (5). Rather, the velocity and temperature fields grow as $\left(u_{i}, \theta\right) \sim \exp \left(n_{s} t\right)$. Substitution into $(5 \mathrm{a}-\mathrm{b})$ yields the rate $n_{s}(k)$

$$
2 n_{s}(k)=-(\nu+\chi) k^{2}+\left[(\nu-\chi)^{2} k^{4}+4 g \alpha \beta x(1+x)^{-1}\right]^{1 / 2},
$$

where $x=\left(k_{x}^{2}+k_{y}^{2}\right) / k_{z}^{2}$. Since $n_{s}(k)>0$ for a wide interval of wavenumbers, it follows that when $\operatorname{NLT}(k)=0$, the system is unstable under perturbations, i.e., the kinetic energy grows exponentially in time, which is clearly unphysical. Let us now switch on the non-linear interactions. Their most distinguishing feature is that their integral over all wavevectors is zero (Batchelor, 1953),

$$
\int \operatorname{NLT}(\boldsymbol{k}) d^{3} \boldsymbol{k}=\mathbf{0},
$$

which explains why they are called transfer terms: the NLT do not generate or dissipate energy, they merely spread that which is available (from the external sources) among eddies of different sizes, mostly but not exclusively, from the energy-rich large eddies to the energy-poor small ones. It follows that a stationary state can be achieved only in the presence of non-zero NLT. In summary, we shall write

$$
\begin{aligned}
& N L T=0, \quad \partial / \partial t \neq 0, \\
& N L T \neq 0, \quad \partial / \partial t=0 .
\end{aligned}
$$

After a model for the NLT has been chosen, one can solve Eqs. (5a-b) and determine the stationary energy spectrum. How well that final state compares with experimental data depends of course on how correctly the NLT have been modeled. Let us consider the following choice

$$
\partial / \partial t=0, \quad \operatorname{NLT}_{1}(\boldsymbol{k})_{i}=\nu_{t}(k) k^{2} u_{i}(\boldsymbol{k}),
$$

where $\nu_{t}$ is a turbulent viscosity. Then, Eqs. (5a-b), with $i=3, u_{3}(k)=w(k)$, yield

$$
\left[\nu+\nu_{t}(k)\right]\left[\chi+\chi_{t}(k)\right] k^{4}=g \alpha \beta \frac{x}{x+1}
$$

where, in analogy with the case of turbulent viscosity, $\operatorname{NLT}_{2}(\boldsymbol{k})=\chi_{\boldsymbol{t}}(k) k^{2} \theta(\boldsymbol{k})$. Equation (10) is a statement about time scales: the right hand side represents the square of the natural frequency of the system, the Brunt-Väisälä frequency, which is required to be equal to the one provided by the non-linear interactions. To solve (10), let us first note that available evidence from different types of turbulence indicates that the so-called turbulent Prandtl number $\sigma_{t}=\nu_{t} / \chi_{t}$ is of order unity or less. Considering further that for large eddies $\nu_{t} \gg \nu$, and introducing $\sigma_{t}, \mathrm{Eq}$. (10) yields

$$
\frac{\chi_{t}}{\chi}=\frac{1}{2}\left[(1+\lambda \Sigma)^{1 / 2}-1\right]
$$


with $\Sigma=4 A^{2}\left(\nabla_{\mathrm{r}}-\nabla_{\mathrm{rad}}\right)$, where the variable $A$ is given by Cox and Giuli (1968, see Eq. 14.70), while the dimensionless constant $\lambda$ (of order unity) is defined as

$$
\lambda=162 \sigma_{t}^{-1} \frac{x+1}{x}\left(k_{0} d\right)^{-4} .
$$

We note that $\chi_{t} / \chi$ is just the convective efficiency, $\Gamma$ (see Cox and Giuli, Eq. 10.106). Multiplying (5b) by $w$ and averaging gives the convective flux $F_{c} / c_{p} \rho=$ $\langle\theta w\rangle$

$$
\langle w \theta\rangle=\beta k_{0}^{-2} \chi^{-1}\left(1+\chi_{t} / \chi\right)^{-1}\left\langle w^{2}\right\rangle,
$$

where the kinetic energy $K$ is given by $2 K=(1+1 / x) w^{2}$ (Canuto and Hartke, 1986). Next, we need to express $K$ in terms of the other variables. This problem has been repeatedly addressed in the turbulence literature and several expressions have been proposed. Among them, the following two are particularly relevant (Canuto et al., 1988)

$$
\nu_{t}=\xi_{3} K^{2} \epsilon^{-1}, \quad \nu_{t}=\xi_{1} \epsilon^{1 / 3} k_{0}^{-4 / 3},
$$

where $\epsilon$ is the rate of energy input per unit mass into the turbulent flow and $\xi_{1}=0.053, \xi_{3}=0.09$. If we write, $F_{c} / c_{p} \rho=\langle w \theta\rangle=\beta \chi_{t}=\beta \chi \Phi$, we have $\epsilon=$ $g \alpha\langle w \theta\rangle=g \alpha \beta \chi \Phi$.

Expressing $K$ in terms of $\nu_{t}$ and $\epsilon, \epsilon$ in terms of $\langle w \theta\rangle$, and the final result in terms of $\Phi$, we obtain, after some algebra

$$
\Phi=2 \frac{x}{x+1} \sigma_{t}^{2}(\lambda \Sigma)^{-1}\left[(1+\lambda \Sigma)^{1 / 2}-1\right]^{3} .
$$

Within factors of order unity, (15) coincides with the MLT expression derived by Gough and Weiss (1976),

$$
\Phi=(9 / 8 \Sigma)\left[(1+\Sigma)^{1 / 2}-1\right]^{3} .
$$

Table 1.

Table 2.

\begin{tabular}{lccc}
\hline$S$ & $\Phi_{\text {MLT }}$ & $\Phi_{1}$ & $\Phi_{1} / \Phi_{\text {MLT }}$ \\
\hline $10^{5}$ & 53 & 115 & 2.17 \\
$10^{6}$ & 173 & 513 & 2.96 \\
$10^{7}$ & 560 & $2.0 \times 10^{3}$ & 3.57 \\
$10^{8}$ & $1.8 \times 10^{3}$ & $7.1 \times 10^{3}$ & 3.94 \\
$10^{9}$ & $5.6 \times 10^{3}$ & $2.4 \times 10^{4}$ & 4.28 \\
$10^{10}$ & $1.8 \times 10^{4}$ & $8.1 \times 10^{4}$ & 4.50 \\
$10^{12}$ & $1.8 \times 10^{5}$ & $8.55 \times 10^{5}$ & 4.75 \\
$10^{14}$ & $1.8 \times 10^{6}$ & $8.76 \times 10^{6}$ & 4.87 \\
$10^{16}$ & $1.8 \times 10^{7}$ & $8.84 \times 10^{7}$ & 4.91 \\
\hline
\end{tabular}

\begin{tabular}{lccc}
\hline$S$ & $K$ & $\Phi_{2}$ & $\Phi_{2} / \Phi_{\mathrm{MLT}}$ \\
\hline $10^{4}$ & $5.3 \times 10^{3}$ & 40 & 2.74 \\
$10^{6}$ & $8.8 \times 10^{5}$ & 1070 & 6.35 \\
$10^{8}$ & $9.8 \times 10^{7}$ & $1.5 \times 10^{4}$ & 8.33 \\
$10^{10}$ & $1.0 \times 10^{10}$ & $1.6 \times 10^{5}$ & 9.09 \\
$10^{12}$ & $1.0 \times 10^{12}$ & $1.7 \times 10^{6}$ & 9.66 \\
$10^{14}$ & $1.0 \times 10^{14}$ & $1.7 \times 10^{7}$ & 9.66 \\
$10^{16}$ & $1.0 \times 10^{16}$ & $1.7 \times 10^{8}$ & 9.66 \\
\hline
\end{tabular}




\section{New models of turbulence}

We shall begin by adopting the EDQNM result for the energy spectrum $E(\boldsymbol{k})$, (Lesieur, 1987)

$$
\left[\partial / \partial t-2 n_{s}(k)\right] E(k, t)=T(k, t),
$$

with the transfer $T(k, t)$ given by

$$
T(k)=\iint d p d q E(q)\left[k^{2} E(p)-p^{2} E(k)\right] a(p, q, k) \tau(k, p, q) .
$$

Here, $a(p, q, k)=\left(x y+z^{3}\right) / q$, and $x, y$, and $z$ are the cosines of the angles opposite $\boldsymbol{k}, \boldsymbol{p}$, and $\boldsymbol{q}$, respectively. The integrations over $p$ and $q$ are such that $\boldsymbol{k}=\boldsymbol{p}+\boldsymbol{q}$. The function $\tau$ is the correlation time scale governing the non-linear interactions. Once $\tau$ is specified, Eq. (17) may be solved for $E(k)$. The convective flux is then computed via

$$
F_{c}=\frac{c_{p} \rho}{g \alpha} \int_{0}^{\infty} 2 E(k)\left[n_{s}(k)+\nu k^{2}\right] d k .
$$

\subsection{First model}

It is well-known from the EDQNM model that the transfer $T(k)$ represents a variety of physical processes among eddies. The most basic process is the energy cascade from large to small eddies which is usually represented by a turbulent viscosity $\nu_{t}(k)$ which, via a closure, must be related to $\theta(k)$. If one approximates $T(k)$ by $(y(k)$ is the mean square vorticity)

$$
T(k) \rightarrow-2 \frac{\partial}{\partial k} y(k) \nu_{t}(k)
$$

one has, after integrating (17) from 0 to $k$, the following equation for $E(k)$

$$
\begin{gathered}
\epsilon(k)=\left[\nu+\nu_{t}(k)\right] \int_{0}^{k} 2 p^{2} E(p) d p, \\
\epsilon(k)=2 \int_{0}^{k}\left[n_{s}(p)+\nu p^{2}\right] d p .
\end{gathered}
$$

The physical interpretation of (21a) is clear: the energy $\epsilon(k)$ injected into the wavenumber interval $[0, k]$ is dissipated partly by molecular viscosity and partly by turbulent viscosity $\nu_{t}$. Canuto et al. (1987) have solved the above equations for $E(k)$ and then calculated the convective flux. The results are presented in Table 1 in which $\Phi_{m l t}$ is given by Eq. (16) and $\Phi_{1}$ is computed using the solution of (21). The variable $S$ is related to $\Sigma$ introduced earlier by $S=(81 / 2) \Sigma$.

\subsection{Second model}

In the second model, we obtain the spectrum $E(k)$ by solving Eq. (17) without any approximation to $T(k)$. Once $E(k)$ is obtained, the flux is again computed using (19). The results are shown in Table 2. 
The turbulent kinetic energy per unit mass $K$ is given in units of $(\chi / \Lambda)^{2}$, where $A$ is the mixing length. The values of $\Phi$ can be represented by the following expression

$$
\Phi=a \Sigma^{m}\left[(1+b \Sigma)^{n}-1\right]^{p}
$$

with $a=24.868, b=9.7666 \times 10^{-2}, m=0.14972, n=0.18931$, and $p=1.8503$. As one can see, in the limit of high convective efficiency, $\Sigma \gg 1$,

$$
\Phi(\text { this model }) / \Phi(\text { MLT }) \approx 10 .
$$

In other words, the new convective flux is up to ten times larger than the MLT value. This result has been recently confirmed by a detailed numerical simulation of turbulent convection (Cabot et al., 1990).

\section{Astrophysical implications}

Since the new model naturally yields a convective flux larger than the MLT, the corresponding value of $\alpha\left(\Lambda=\alpha H_{p}\right)$ will be correspondingly smaller and thus more in accord with the basic tenets of incompressibility adopted in all the previous models. Since $F_{c} \propto \Lambda^{2} \propto \alpha^{2}$, we estimate that

$$
\alpha_{\text {new }} \sim \alpha_{\text {MLT }} /(5-8)^{1 / 2}
$$

Since $\alpha_{\mathrm{MLT}}=1.4$, we predict $\alpha_{\text {new }}=0.5-0.6$. Indeed, detailed evolutionary studies indicate that $\alpha_{\text {new }}=0.7$ (Canuto and Mazzitelli, 1991). It may also be noted that the new larger convective flux may have important consequences in helioseismology (L. Paterno, private communication) and in the determination of the age of globular clusters.

\section{References}

Batchelor, G.K.: 1953, The Theory of Homogeneous Turbulence, Cambridge Univ. Press Böhm-Vitense, E.: 1958, Zeitschr. Astrophys. 46, 108

Cabot, W., Hubickyj, O., Pollack, J.B., Cassen, P., Canuto, V.M.: 1990, Geophys. and Astrophys. Fluid Dyn. 53, 1

Canuto, V.M., Goldman, I.:1985, Phys. Rev. Letters 54, 430

Canuto, V.M., Goldman, I., Chasnov, J.: 1987, Phys. Fluids 30, 3391

Canuto, V.M., Hartke, G.J.: 1986, Astron. Astrophys. 168, 89

Canuto, V.M., Goldman, I., Chasnov, J.: 1988, Astron. and Astrophys. 200, 291

Canuto, V.M., Mazzitelli, I.: 1991, Astrophys. J., (in press).

Cox, J. P., Giuli, R. T.: 1968, Principles of Stellar Structure, Gordon and Breach

Gough, D.O., Weiss, N.O.: 1976, Mon. Not. Roy. Astron. Soc. 176, 589

Kraichnan, R.H.: 1964, Phys. Fluids 7, 1048

Lesieur, M.: 1987, Turbulence in Fluids, M. Nijhoff Publ.

Ledoux, P., Schwarzschild, M., Spiegel, E.A.: 1961, Astrophys. J., 133, 184

Nakano, T., Fukushima, T., Unno, W., and Kondo, M.: 1979, Publ. Astron. Soc. Japan 31, 713

Yakhot, V. and Orszag, S.A.: 1986, Jour. of Sci. Comp. 1, 3

Yamaguchi, S.: 1963, Publ. Astron. Soc. Japan 15, 412 